\title{
Exposición a inundaciones en Moravia, San José, Costa Rica
}

\section{Exposure to floods in Moravia, San José, Costa Rica}

\author{
Mario Fernández, Arce \\ Ileana Méndez Ocampo ${ }^{2}$ \\ Rudy Muñoz Jiménez ${ }^{3}$
}

Fecha de recepción: 20 de noviembre de 2017

Fecha de aceptación: 3 de setiembre de 2018

\begin{abstract}
Resumen
Se inspeccionaron los valles de los ríos de Moravia para identificar y cartografiar los asentamientos irregulares así como las zonas inundables. Esto se hizo porque algunas áreas protegidas de los ríos, tanto en zonas rurales como urbanas, están siendo invadidas por el ser humano y la mayoría de las veces, los asentamientos resultantes de la invasión se convierten en sitios de alto riesgo para la vida. Al identificar tales lugares se pretende crear las bases para iniciar la gestión de los riesgos en los sitios donde la población está expuesta a los peligros. El estudio requirió investigación bibliográfica, trabajo de campo y análisis de fotografías. Las principales conclusiones son: 1 - Hay 9 casos, identificados en este trabajo, de invasión a valles de ríos y quebradas en el cantón, la mayoría de ellos están ubicados a lo largo del río Ipís. 2 - El ser humano tiende a generar riesgos que no quiere al no aplicar la normativa existente para prevenir desastres. 3 - En oposición al patrón conocido, gente de clase media está viviendo en zonas de alto riesgo.

Palabras claves: Inundaciones, Ríos, Moravia, Amenaza, Vulnerabilidad
\end{abstract}

\begin{abstract}
Humans have invaded some river's protected areas, both in rural and urban zones, turning them, most of the times, into high-risk areas. The exploration of the river valleys of Moravia allowed the confirmation and location of settlements and flood-prone areas. Such identification created the groundwork to risk management in order to protect the population from health hazards. This study required bibliographical research, fieldwork and photograph analysis. The main conclusions are: 1 - This research identified 9 cases of invasion to valleys of river and streams in Moravia, most of them are located along the Ipis river, 2 - Human activity generates undesired risk because people do not follow the normative to prevent disasters, 3 - In opposition to the known pattern, Moravia's middle-class people are living in high-risk areas.
\end{abstract}

KeyWords: floods, rivers, Moravia, hazard, vulnerability

1. Universidad de Costa Rica, Escuela de Geografía y Programa PREVENTEC, mario.fernandezarce@ucr.ac.cr

2._Municipalidad de Moravia, San José, Costa Rica, imendez@moravia.go.cr

3._Universidad de Costa Rica, Estudiante de la Escuela de Geografía, rudy40_4@ hotmail.com

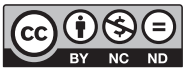

Rev. Reflexiones 98 (1) Enero-Junio, 2019: 7-21 


\section{Introducción}

Se realizó un estudio sobre inundaciones en el cantón Moravia (Figura 1). Durante la investigación se hizo una inspección de los valles de los ríos para comprobar que ellos han sido invadidos por asentamientos humanos, lo cual se documentó con fotografías. Lo anterior es importante y necesario porque las invasiones a las zonas de protección de flujos de agua fomentan el problema de las inundaciones y, por ende, la afectación a seres humanos. Simultáneamente, se revisaron informes de situación sobre incidentes ocurridos en el cantón para conocer con detalle los puntos donde han ocurrido desbordamientos e inundaciones. Los sitios invadidos observados y los inundados fueron descritos y cartografiados. Finalmente, se hicieron extractos de tres leyes nacionales que se refieren a restricciones para vivir en zonas de alto riesgo y en áreas protegidas del cauce de los ríos, con el fin de demostrar que en los sitios inundados no tendría que haber asentamientos humanos y que, por tanto, no tendrían que haber inundaciones con afectación humana en ellos.

Figura 1

Área de estudio

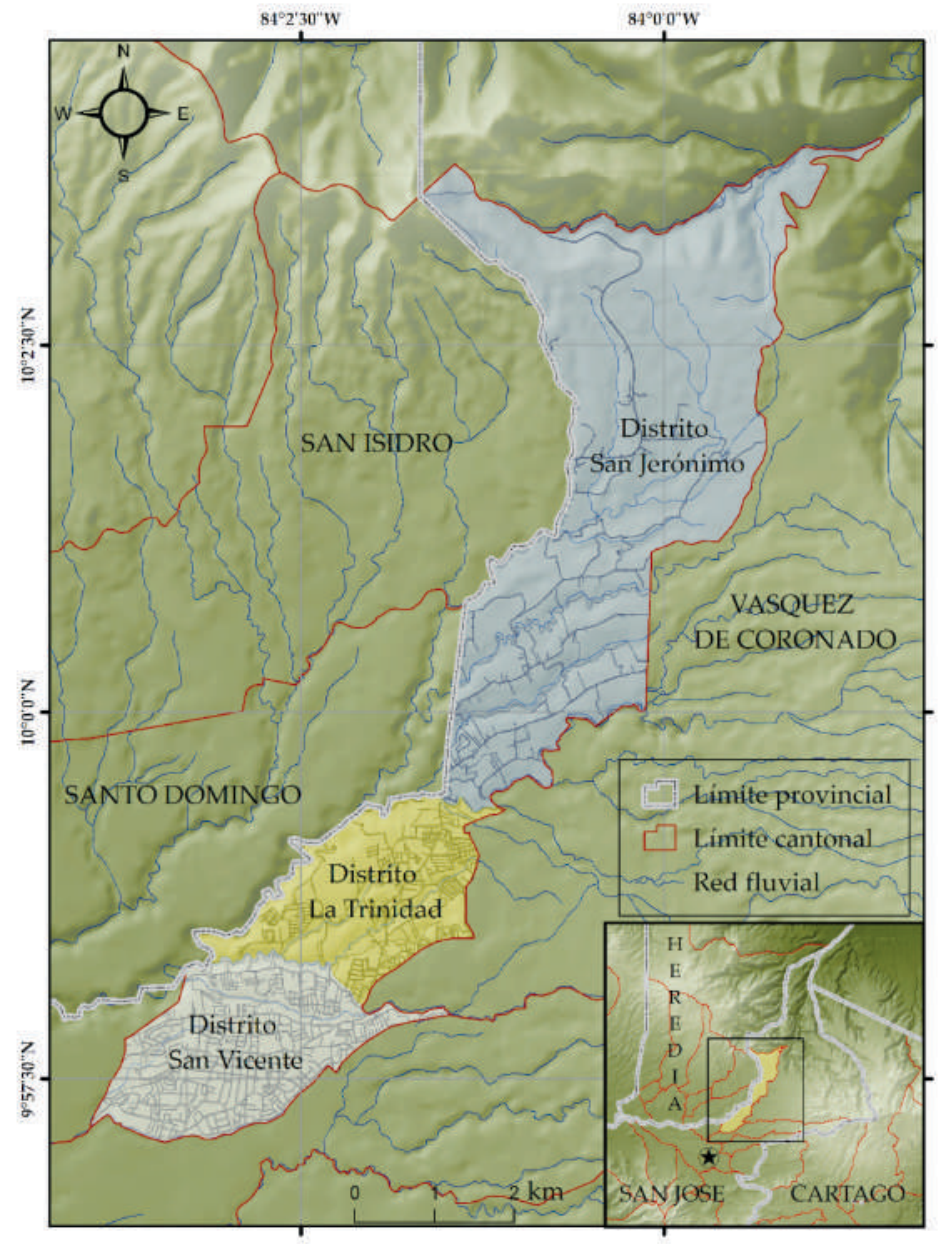

Fuente: Elaboración propia.

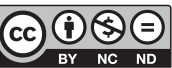

Rev. Reflexiones 98 (1) Enero-Junio, 2019: 7-21, E-ISSN: 1659-2859 
Las áreas de protección del cauce de los ríos se han convertido en un atractivo para los procesos de crecimiento irregular que ponen en peligro vidas humanas, la cubierta forestal y equilibrio ecológico, los suelos y las aguas subterráneas. En Costa Rica, al igual que en muchos países latinoamericanos, hay sectores de la población que no tienen recursos económicos ni acceso a crédito para obtener una vivienda en los mercados formales, por lo que dichos sectores no tienen más alternativa que buscar lotes baratos y, generalmente, no aptos para vivir. Como consecuencia de esto, terminan viviendo en zonas de alto riesgo, donde su vida corre peligro.

El objetivo del estudio fue identificar las zonas de protección invadidas y las áreas inundables del cantón Moravia, a fin de gestionar adecuadamente el riesgo que implica vivir en esas zonas de alta peligrosidad. Todo asentamiento ubicado dentro del valle de un río está en un terreno de alta pendiente o en una zona propensa a inundación, por lo que se requiere un plan para reducir el riesgo asociado a un deslizamiento o un desbordamiento de río. Pero sin estudios que pongan en evidencia la problemática y muestren los sitios de posible afectación es difícil iniciar planes que busquen mitigar y prevenir.

Se recurrió a una revisión bibliográfica para conocer las áreas inundables del cantón. El trabajo de investigación se orientó fundamentalmente a un estudio de campo durante el que se visitaron los asentamientos irregulares para conocerlos y ubicarlos. El objetivo de las visitas fue identificar con GPS los sitios que estuvieran bajo el borde superior del valle del río.

\section{Metodología}

Este estudio se basó en trabajo de campo durante el periodo 2015-2017. Las visitas de campo se hicieron para observar directamente la ocupación de las áreas restringidas por las leyes nacionales (mayor detalle en la sección sobre Normativa) y determinar el grado de amenaza de las comunidades expuestas. Para ello, recorrimos los ríos con GPS y equipo audiovisual que permitiera documentar los casos. Era de vital importancia verificar si el asentamiento estaba bajo el borde superior del valle y si se ubicaba en sitio de alta pendiente. Las zonas de interés fueron debidamente identificadas tanto en el campo como en el mapa. En las expediciones se tomaron fotografías desde el suelo (en las fechas 22-92015 y 11-7-2016) que permiten demostrar la invasión del proceso urbanístico en los valles.

La investigación requirió revisión bibliográfica por lo que se consultaron publicaciones en libros, revistas y periódicos sobre incidentes y acontecimientos de origen natural o antrópico en comunidades ubicadas en los valles de los ríos de Moravia, así como informes de situación y técnicos, elaborados por funcionarios de entidades públicas, particularmente aquellos realizados por miembros de la Comisión Nacional de Prevención de Riesgos y Atención de Emergencias (CNE). Uno de los autores ha tenido que atender las últimas inundaciones que se han presentado en el cantón, lo que permitió recopilar información valiosa. De esta etapa del trabajo surgió el mapa de sitios inundables del área de estudio.

\section{Resultados}

\section{Áreas invadidas}

El valle del río Ipís y otras quebradas de Moravia han sido invadidos por el ser humano como se muestra en las siguientes figuras (fotos). Los sitios mostrados en cada foto se presentarán como un

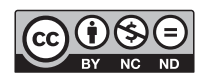


punto en el mapa de la Figura 7). En su mayoría es gente pobre la que ha invadido pero también hay personas de clase media viviendo en urbanizaciones y condominios de gran valor económico. Todas las imágenes evidencian que las casas están en una zona de alta pendiente lo cual es evidente a simple vista o es evidenciado por los grandes pilotes que sostienen las casas.

El sitio mostrado la Figura 2 (F1) se encuentra ubicado en Calle San Francisco de la Trinidad de Moravia. La imagen fue tomada desde Calle El Moral y en ella se puede apreciar parte de un cafetal, un camino que desciende a un nivel inferior, casas humildes en la parte superior plana (inicio del valle y de la pendiente), un conjunto de casas sencillas en ladera y el Condominio Fuente de Vida (a la izquierda, estructura de color blanco con árboles delante). Esta última obra ha pasado por un proceso ante el Ministerio de Salud y el Departamento de la Dirección Técnica Operativa de la Municipalidad de Moravia por irregularidades en sus planos.

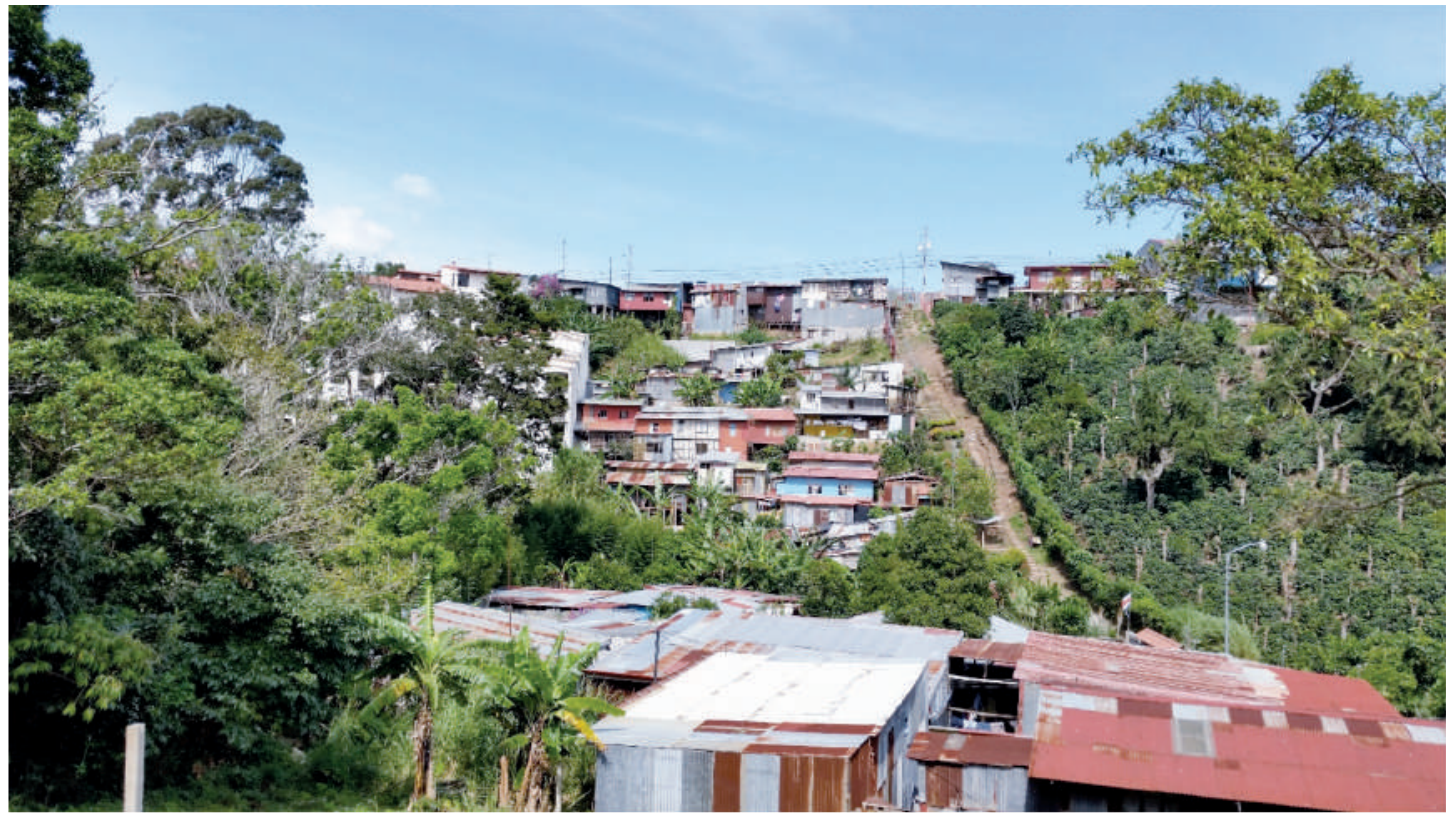

Fuente: Fotografía de Mario Fernández Arce, fotografía tomada el 15-12-2015.

Todas las estructuras antes mencionadas están en la ladera derecha de la quebrada San Francisco (aguas abajo). En la pendiente opuesta hay un conjunto de casas humildes de las cuales se observan principalmente los techos (parte inferior de la fotografía). Aunque no se aprecia muy bien, el camino tiene gradas de concreto y es la vía por la cual salen las personas que viven en la parte más baja del cañón. Ese camino atraviesa la quebrada (hay puente peatonal) de manera que las personas pueden transitar en uno y otro sentido. Llama la atención la coexistencia de un asentamiento de clase muy baja y un condominio de clase media en el valle en cuestión. Ni un desarrollo ni el otro deben estar en esa zona.

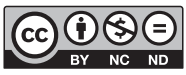


El área mostrada en la Figura 3 (F2) está ubicada en la margen izquierda, aguas abajo, del río Ipís, en la zona limítrofe entre el centro urbano de San Vicente y la comunidad de La Isla, a la altura del puente del vecindario conocido como Barro de Olla. La línea de árboles a uno y otro lado del cauce delimita el ancho del valle por lo que, evidentemente, el grupo de viviendas mostradas está dentro del mismo y sobre una terraza (parte plana elevada sobre el cauce) de la pendiente del río. La vulnerabilidad humana por aspectos físicos es alta en este sitio.

Figura 3

Asentamientos humanos en el río Ipís, vecindario Barrio de Olla (F2)

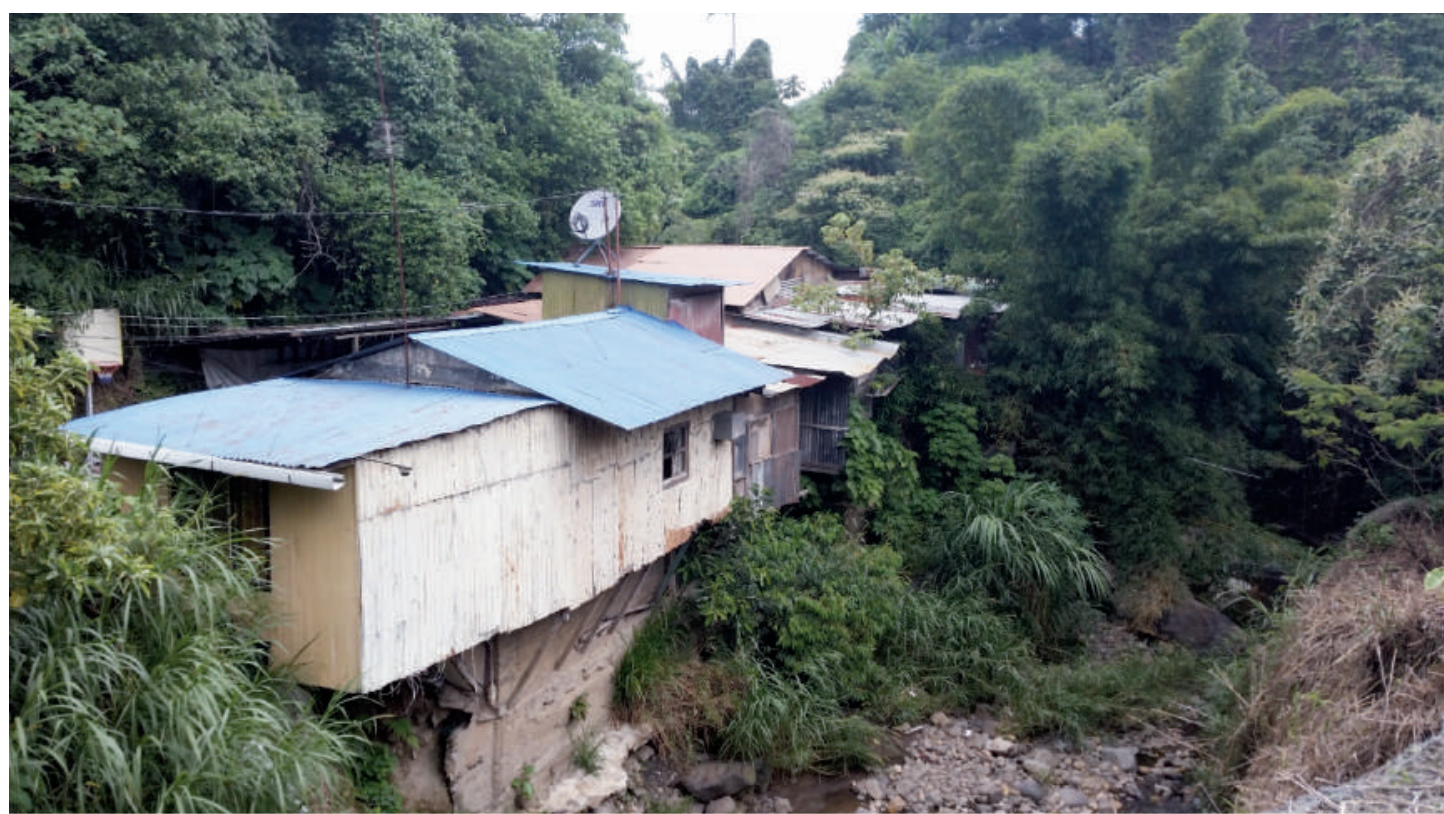

Fuente: Fotografía de Mario Fernández Arce, fecha de la fotografía: 22-09-2015.

Se nota que parte de la primera casa no está apoyada en la superficie del terreno y que la pared natural del río debajo de la casa ha sido reforzada con un revestimiento de concreto, muy probablemente para evitar la erosión y el eventual desprendimiento de la obra. Pero esas medidas podrían ser insuficiente para evitar el daño. El poder de penetración del agua, y por tanto su poder destructivo, es grande y podría superar la resistencia del revestimiento. Si el material de la terraza fuera de roca sana, dura y resistente sería muy difícil que el río desestabilizara las casas, pero bajo ellas existe un suelo y no roca, lo que podría favorecer la infiltración de agua entre el suelo y el concreto y con ello, el debilitamiento de la base de las viviendas y el deslizamiento del suelo. Corrientes de gran magnitud, provocadas por picos o máximos de precipitación, podrían comprometer la estabilidad de las casas mostradas en la Figura 3.

La vivienda de la Figura 4 (F3) está ubicada en la margen derecha del río Ipís, en el vecindario La Isla. Es una más de un conjunto de casas construidas en una estrecha franja de terreno entre la calle pública y el flujo de agua. Se podría decir que la acera es la única obra admisible en ese espacio pues a partir de ella inicia el valle del río y su respectiva pendiente.

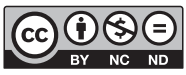


Figura 4

Vivienda cerca al río Ipís, La Isla (F3)

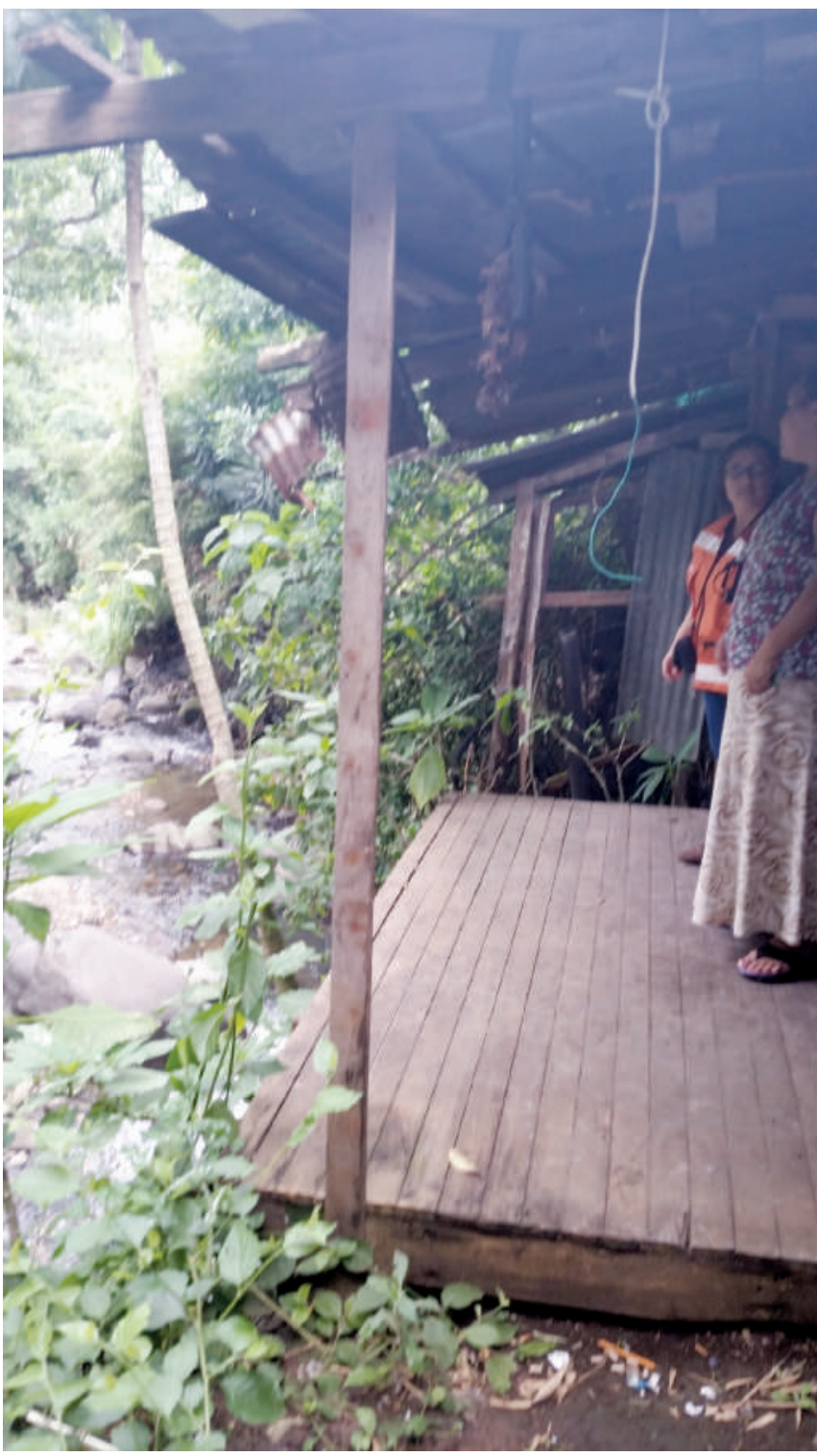

Fuente: Fotografía de Mario Fernández Arce, fecha de la fotografía: 22-09-2015.

El problema de ese hogar en particular es que la casa, aparte de estar en la angosta ladera del río, está en una curva del mismo, justamente después de un tramo rectilíneo de orientación noreste-suroeste. Lo grave de ello es que un gran torrente de agua podría impactar violentamente las bases de la vivienda, erosionar el suelo y derribar la estructura.

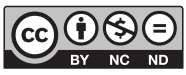

Rev. Reflexiones 98 (1) Enero-Junio, 2019: 7-21, E-ISSN: 1659-2859 
Quizá no sea la caída de la casa en el agua lo que más preocupa, ya que los ocupantes de la misma están en constante vigilancia, sino la angustia de quienes estén en dicha residencia cuando llueve. En una noche de lluvias intensas y prolongadas sería difícil dormir con tranquilidad es ese hogar. Las gestiones realizadas para solventar esta situación incluyen la información y preparación para una respuesta que salve vidas, pero lo ideal es que estas personas dejen el lugar. Sin embargo, el municipio no tiene recursos para otorgar viviendas y las personas, como ya se ha mencionado, son de escasos recursos.

El conjunto de viviendas observado en la Figura 5 (F4) está en el vecindario conocido como La Isla, al sur de la urbanización Lomas de Moravia. Se trata de un grupo de viviendas de familias de escasos recursos que, como se aprecia en la fotografía, están en una ladera, más abajo de una superficie plana que marca el inicio del valle del río. Dos elementos que dan una idea de la alta pendiente del lugar son el camino con gradas y los apoyos de la casa de zinc que ocupa el plano central de la fotografía. Las gradas de este sector son similares a las del punto F1 y al igual que aquellas, están hechas de concreto para dar estabilidad al suelo y evitar que se deteriore el camino.

Figura 5

Asentamiento humano en el margen derecho del río Ipís, en La Isla (F4)

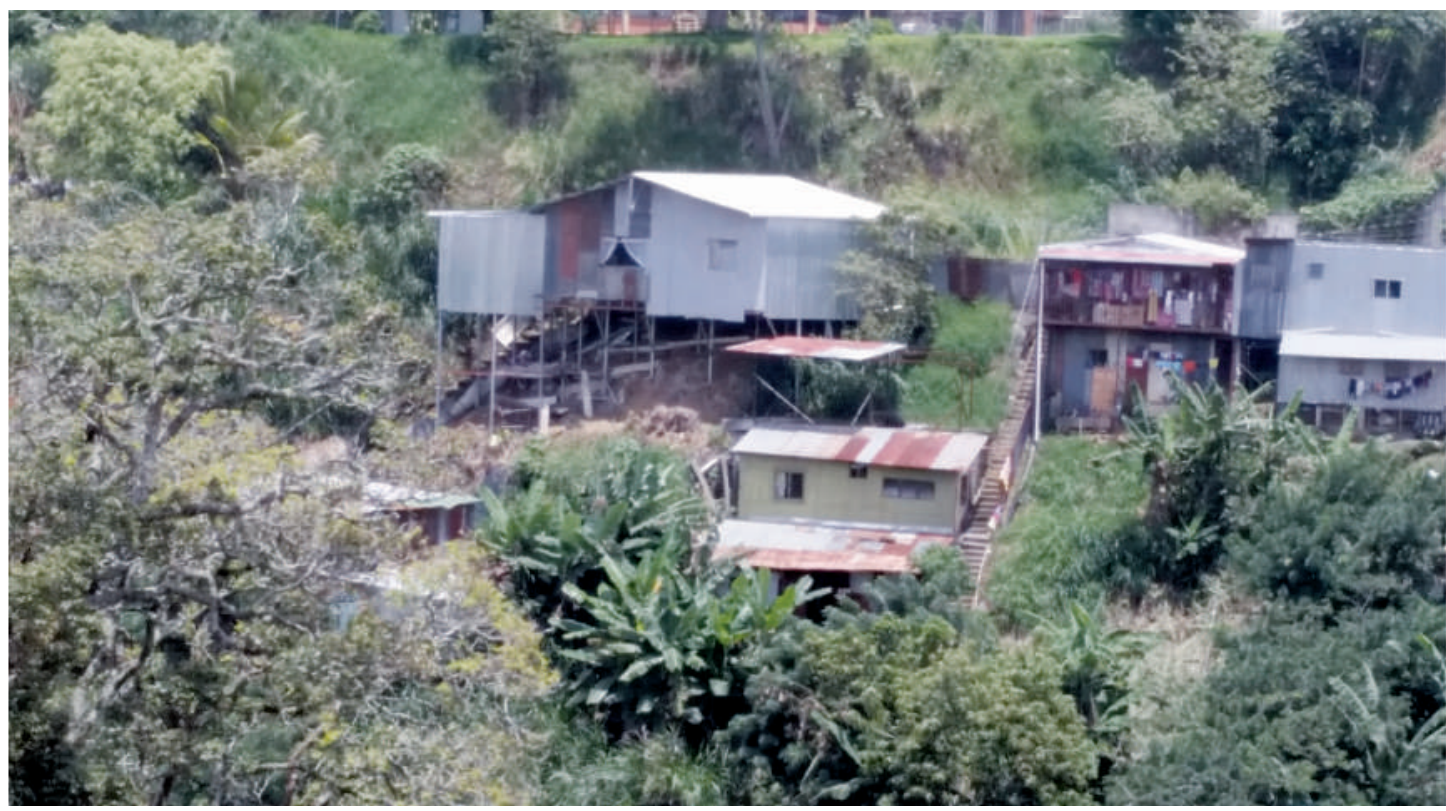

Fuente: Fotografía de Mario Fernández Arce, fecha de la fotografía: 22-09-2015.

El otro elemento que resalta la alta pendiente en la que se encuentran las construcciones son las bases de las viviendas. Se nota en algunos casos que estas son altas y delgadas y sirven para darle nivel y horizontalidad a la estructura. Esa es la manera de compensar el desnivel generado por la alta pendiente. Pero tal práctica es un inconveniente en un país como Costa Rica, donde la sismicidad es alta, al igual que la precipitación, procesos naturales que disparan los deslizamientos. En laderas donde las casas están escalonadas en diferentes niveles, pequeños deslizamientos en las partes altas pueden impulsar unas viviendas contra otras incrementando con ello la destrucción.

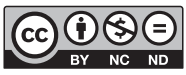

Rev. Reflexiones 98 (1) Enero-Junio, 2019: 7-21, E-ISSN: 1659-2859 
A propósito de daños, después de las lluvias del lunes 8 de mayo de 2017 se dio un deslizamiento cerca del sitio mostrado en la Figura 5. Cuatro viviendas fueron afectadas y como consecuencia, hubo que evacuar dichas casas y trasladar 23 personas a un albergue ubicado en la Isla. Entre las personas movilizadas había 11 niños con edades entre 3 y 11 años.

La situación del área mostrada en la Figura 6 (F5) es muy similar a la de la Figura 4. Se trata de una casa de 2 niveles ubicada en la margen derecha del río Ipís, la segunda después del puente sobre la vía que conduce de San Rafael hacia el sector Este de la Isla. Dicha vivienda está apoyada sobre delgadas y, aparentemente, débiles bases o pilotes de madera. En general, la condición y la resistencia de la obra son deficientes. Según la conversación sostenida con una habitante, en el momento de la visita allí vivían una adulta mayor, dos menores de edad, dos mujeres adultas, un joven, la madre de los niños y una joven con autismo. La Municipalidad de Moravia, por medio de su Oficina de Gestión del Riesgo y Atención de Emergencias Cantonales realizó un informe para dicho caso (Méndez, 2016, Chaves, 2016b).

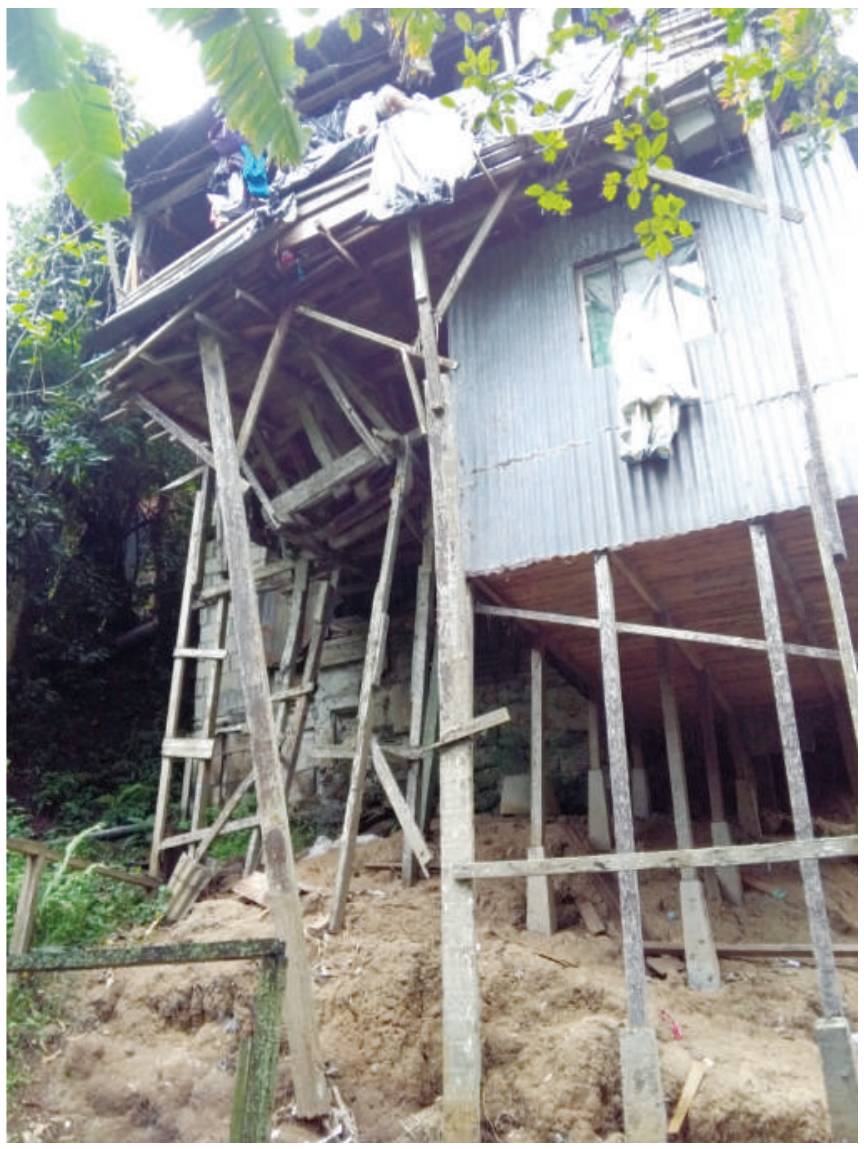

Fuente: Fotografía de Mario Fernández Arce, fecha de la fotografía: 11-07-2017.

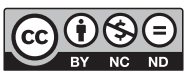


Es difícil que el agua del río llegue siquiera al nivel inferior de la casa pero eso no descarta que la misma pueda terminar en el cauce. Lluvias intensas o la ocurrencia de un temblor fuerte, cercano a Moravia, podrían afectar la estabilidad del suelo en el que está la obra; y por ende, la de la casa. Los esfuerzos hechos para paliar esta situación incluyen charlas informativas sobre la condición de vulnerabilidad de quienes viven en dicha casa y advertencias de no usar la parte posterior de la misma, principalmente a la adulta mayor quién tiene problemas auditivos. La municipalidad recomendó desocupar dicha vivienda e informó a la CNE y al IMAS para que hicieran las valoraciones del caso y ayudaran desde su competencia a esta familia. La CNE propuso que un ingeniero valorara la vivienda pero la familia es de escasos recursos por lo que no pueden contratar los servicios del profesional indicado.

\section{Áreas inundables}

Hasta el momento, las inundaciones de Moravia no han causado mayores daños, pero requieren atención por cuanto la exposición física del ser humano a ellas podría estar aumentando. En la Tabla 1 se indican los sitios afectados por inundaciones en el cantón Moravia según los datos encontrados.

Tabla 1

Sitios afectados por inundaciones en Moravia.

\begin{tabular}{llll}
\hline Punto & Fuente & Fecha & Lugar \\
\hline & & & Coordenadas planas \\
PI1 & DPM-INF-059-2007 & $02-02-2007$ & Los Sitios: 495743 FN / 1102751 FW \\
PI2 & DPM-INF-0571-2008 & $30-04-2008$ & San Blas: 495969 FN / 1101945 FW \\
PI3 & DPM-INF-1806-2008 & $31-12-2008$ & San Blas: 496201 FN / 1102102 FW \\
PI4 & DPM-INF-0223-2009 & $04-03-2009$ & San Martín: 492783 FN / 1100778 FW \\
PI5 & DPM-INF-0936-2010 & $15-03-2011$ & La Trinidad: 497177 FN / 1103726 FW \\
PI6 & DPM-INF-0872-2012 & $30-01-2013$ & Barrio Corazón de Jesús: 493903 FN / 1101958 FW \\
PI7 & IAR-INF-0065-2016 & $21-01-2016$ & Lomas de Cabiara: 497490 FN / 1103738 FW \\
\hline
\end{tabular}

Fuente: Elaboración propia.

En la Figura 7 se muestran tanto los puntos desde donde se tomaron las fotos antes mostradas (F1 a F5) como los puntos inundables listados en la Tabla 1.

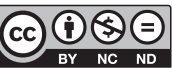


Figura 7

Puntos afectados por inundaciones en Moravia

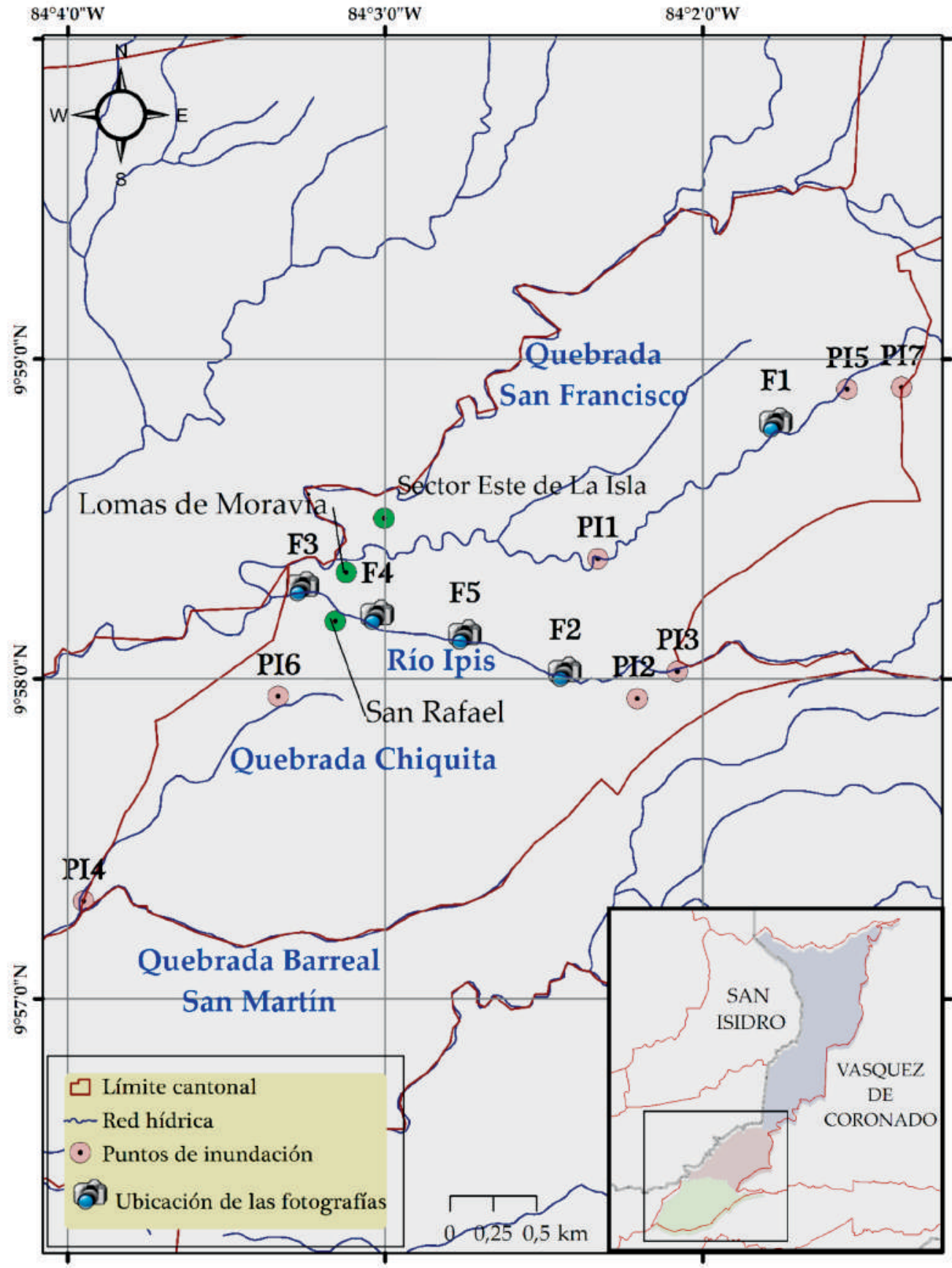

Fuente: Elaboración propia. 
El punto PI1 es un sitio que fue afectado por una inundación en febrero del 2007. En esa ocasión se reportó daño a una casa de Los Sitios ubicada en la zona de protección de la Quebrada San Francisco, la cual había sido falseada por erosión fluvial (Bonilla, 2007). Según tal autor, esa, otras viviendas y un muro para protegerlas no guardan la distancia de los $10 \mathrm{~m}$ de protección que establece la ley.

El PI2 está en San Blas de Moravia. Durante la estación lluviosa del 2008, la parte trasera de la escuela de San Blas se inundó, probablemente por incapacidad hidráulica de las alcantarillas cercanas (Barahona, 2008). En la parte afectada hay una salida de alcantarilla. La pendiente del terreno facilita la llegada de agua a las instalaciones.

En el punto PI3 hay viviendas en la margen izquierda del río Ipís, a menor altura que el nivel de la carretera y dentro del área de dinámica del río (Sjöbohm, 2008). Ellas están en una pequeña terraza que podría ser erosionada y socavada por aumento de la carga hidráulica. En tales condiciones, las casas podrían verse afectadas por las crecidas del río producto de las fuertes lluvias y el agua proveniente de actividad antrópica. Según Sjöbohm (2008) estas residencias requieren reubicación.

El sitio PI4 se encuentra entre las quebradas Chiquita y Barreal San Martín, como se puede apreciar en la Figura 7. Esas quebradas reciben aguas servidas y por ello, aumenta rápidamente su caudal durante fuertes aguaceros. En el lugar hay propiedades en la zona de protección del cauce (casas) y obras de protección como muros y tapias. Algunas viviendas se encuentran a escasos metros de altura con respecto al cauce. Allí ocurren desbordamientos y erosión fluvial hacia la margen de la quebrada Barreal.

En el 2009, una casa fue afectada por las inundaciones (Sjöbohm, 2009). Este no es el único punto donde la Quebrada Chiquita ha ocasionado problemas de erosión fluvial a lo largo de su trayecto; la Comisión Nacional de Prevención del Riesgo y Atención de Emergencias ha identificado otros puntos afectados como el sector del Parque Infantil, a la altura de la urbanización Los Robles, antes y después del puente de Calle Umaña. En ese mismo lugar se ubican los Condominios Topacio (Chaves, 2017).

En la Trinidad de Moravia (PI5) hubo inundaciones en el 2010 (Chaves, 2010). Viviendas ubicadas en la zona de protección de la Quebrada San Francisco fueron totalmente anegadas después de intensas lluvias en setiembre. Este evento afectó a 4 viviendas y a un total de 12 personas. La zona afectada es inhabitable por vulnerabilidad física y sólo sirve para protección del cauce del río. A pesar de esto, existen en el sitio viviendas construidas de forma ilegal y sin permisos de la Municipalidad.

Una situación adversa ocurrió en un terreno ubicado en el Barrio Corazón de Jesús, al este del Residencial Los Colegios Norte (PI6). El problema fue ocasionado por un flujo de lodo que viajó a través de un canal natural abierto que recibe tanto agua de lluvia como de viviendas (Campos, 2012). La principal consecuencia del evento fue la anegación de una vivienda, la cual quedó severamente afectada y con problemas estructurales. Dicha casa está en un terreno propenso a erosión y filtración de aguas. En este caso, mucho del problema se debe a que la vivienda se ubica pendiente abajo y al quedar el canal obstruido, el agua fluye por la pendiente y afecta lo que haya abajo.

Finalmente, otro punto con problemas por inundación está en el sector de Lomas de Cabiara de la Trinidad de Moravia (PI7), donde se reportó una vivienda en la zona de protección de la Quebrada Jesús, afluente de la quebrada San Francisco en 2016. En ese año, tal estructura estaba a desnivel con respecto a la vía principal, lo que favorecía que el agua de escorrentía ingresara a la propiedad. La estructura de la vivienda estaba en mal estado y la base de la cocina estaba siendo erosionada por la quebrada (Chaves, 2016a).

Observando la Figura 7 se concluye que en los sitios fotografiados no ha habido problemas de inundación hasta la fecha y ello se debe a que están relativamente lejos (a mayor altura) de los flujos

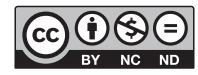


de agua. Algunos de ellos podrían ser afectados si el caudal sube desproporcionadamente a raíz de eventos máximos de precipitación. Pero también podría haber inundaciones en ellos si el proceso de construcción de viviendas sigue avanzando hacia el río. Las inundaciones han ocurrido en sitios más planos donde el nivel del agua de las quebradas y ríos está más cercano a las casas. Esos lugares suelen ser pequeñas llanuras de inundación, muy atractivas para el establecimiento de asentamientos humanos.

\section{Normativa inefectiva para evitar la exposición}

Los asentamientos humanos que hoy día están dentro de los valles de las quebradas y ríos de Moravia no estarían allí si se hubiese seguido el procedimiento adecuado para construir las obras y se hubiese aplicado tres leyes primordiales a saber: La Ley Nacional de Prevención de Riesgos y Atención de Emergencias, La Ley Forestal y La Ley Orgánica del Ambiente. Estas tres leyes contribuyen a evitar que la ciudadanía se exponga a las amenazas. A continuación se citan tres leyes importantes que riñen con la situación de los asentamientos antes mencionados.

\section{La Ley Nacional de Prevención de Riesgos y Atención de Emergencias}

Esta es la ley No. 8488 del 2005, la más importante para el país en materia de gestión de riesgos. Su Artículo 14 se refiere a las competencias de la Comisión Nacional de Prevención de Riesgos y Atención de Emergencias (CNE) y en el inciso c de ese artículo la ley dice textualmente:

Dictar resoluciones vinculantes sobre situaciones de riesgo, desastre y peligro inminente, basadas en criterios técnicos y científicos, tendientes a orientar las acciones de regulación y control para su eficaz prevención y manejo, que regulen o dispongan su efectivo cumplimiento por parte de las instituciones del Estado, el sector privado y la población en general. Los funcionarios de los órganos y entes competentes para ejecutar o implementar tales resoluciones vinculantes, en ningún caso, podrán desaplicarlas. A las personas físicas o jurídicas, públicas o privadas, que edifiquen o usen indebidamente zonas restringidas mediante estas resoluciones vinculantes, se les aplicará la obligación de derribar o eliminar la obra, conforme al artículo 36 de esta Ley. (p.13)

\section{La Ley Forestal}

La ley Forestal de Costa Rica No. 7575 del año 1996, en su capítulo IV, Artículo 33 sobre áreas de protección, inciso b, dice textualmente que se declara área protegida:

una franja de quince metros en zona rural y de diez metros en zona urbana, medidas horizontalmente a ambos lados, en las riberas de los ríos, quebradas o arroyos, si el terreno es plano, y de cincuenta metros horizontales, si el terreno es quebrado. (p. 16)

El artículo 33 de la Ley Forestal lo que busca es proteger el agua y los ecosistemas asociados a los ríos, los lagos y los embalses y no directamente a las personas del embate de las amenazas, pero la protección ocurre de manera indirecta. Si se respetaran esas zonas no habría tantos asentamientos en zonas inundables ni en laderas de los cañones de los ríos donde pueden ocurrir mortales deslizamientos.

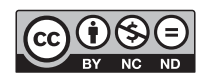




\section{La Ley Orgánica del Ambiente}

La Ley orgánica del ambiente No. 7554 de 1995 en su artículo 28 dice textualmente:

Es función del Estado, las municipalidades y los demás entes públicos, definir y ejecutar políticas nacionales de ordenamiento territorial, tendientes a regular y promover los asentamientos humanos y las actividades económicas y sociales de la población, así como el desarrollo físico-espacial, con el fin de lograr la armonía entre el mayor bienestar de la población, el aprovechamiento de los recursos naturales y la conservación del ambiente. (p. 15)

Respecto a los fines de la ley, el artículo 29, inciso a., dice que se considerará un fin:

Ubicar, en forma óptima, dentro del territorio nacional las actividades productivas, los asentamientos humanos, las zonas de uso público y recreativo, las redes de comunicación y transporte, las áreas silvestres y otras obras vitales de infraestructura, como unidades energéticas y distritos de riego y avenamiento. (p. 16)

\section{Incumplimiento de la Normativa}

Todo asentamiento humano ubicado dentro del valle de un río incumple la normativa existente sobre zonas de protección del cauce de los ríos. Por tanto, los asentamientos y las viviendas de Moravia ubicadas en dichas áreas no se ajustan a leyes existentes ni a la planificación y ordenamiento del territorio. Se desconoce cuándo y en cuáles circunstancias llegaron esas personas a vivir en esos sitios. En términos generales, la invasión puede deberse a: realización de obras antes de la existencia de la ley, permisos de construcción otorgados, corrupción, prevaricato o al desarrollo de asentamientos informales.

Si se alega que la obra fue antes de la Ley Forestal 7575 del año 1995, el argumento podría ser inadmisible porque según La Fiscalía Adjunta Agrario Ambiental del Ministerio Público de Costa Rica (Boletín N 6: El Delito de Invasión de Áreas de Protección), existe normativa que salvaguarda el área de protección desde 1939 y al menos desde 1969 existe un delito para sancionar a quien invada dichas áreas. En algunos casos la invasión puede darse porque el solicitante de un permiso oculta datos sobre la sede real del lote donde espera construir. Si ese es el caso, la Municipalidad puede incurrir en un error y otorgar el permiso por imprecisión de los requisitos. En otros casos, la compasión puede llevar al otorgamiento del permiso, el funcionario municipal sabe que el terreno no es apto pero se compadece de la necesidad del solicitante y concede el permiso aun cuando vaya en contra de la ley.

\section{Conclusiones}

Valles de los ríos y quebradas del cantón de Moravia han sido invadidos por el ser humano y sus actividades, principalmente, el del río Ipís y la quebrada San Francisco. Se identificaron cinco asentamientos sobre el río Ipís, de los cuales dos son de gran importancia por la pendiente y la vulnerabilidad física, ya que contienen construcciones en regulares o malas condiciones.

Otro de los casos críticos se encuentra en la quebrada San Francisco (punto PI5), donde hay un precedente de arrastre de las viviendas por la corriente de agua de dicha quebrada. En la actualidad las personas siguen viviendo en ese mismo lugar y ha aumentado la cantidad de construcciones en dicho sitio.

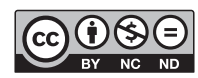


En total, existen 9 casos de ubicaciones en valles de ríos y quebradas, la mayoría sobre el río Ipís, pero también hay asentamientos en las quebradas San Francisco, Jesús y Chiquita. El mayor número de las casas ubicadas en el valle de los ríos es de familias de escasos recursos pero también se observaron casas de gran valor en las zonas de protección, de familias de clase media, como el condominio Fuente de Vida que está en el valle de la quebrada San Francisco. Esto indica que en Moravia no sólo las personas en condición de pobreza están en zonas de alto riesgo, sino que el intenso proceso de urbanización ha llevado a personas de buen ingreso económico a sitios peligrosos.

Las inundaciones ocurren en los distritos del sur (San Vicente y La Trinidad) es decir, en zonas urbanas. El sector norte es más rural, montañoso y de relieve alto y abrupto lo que coadyuva para que en él prácticamente no haya afectación por desbordamiento de ríos y quebradas.

Los asentamientos y las viviendas de Moravia ubicados en áreas restringidas incumplen leyes existentes y no se ajustan a procesos de planificación y ordenamiento del territorio. En términos generales, la invasión puede deberse a: realización de obras antes de la existencia de la ley, permisos de construcción otorgados inadecuadamente o a la ilegalidad.

El ser humano tiene diferentes roles en el desarrollo de asentamientos en los valles de los ríos. Los inversionistas o desarrolladores de proyectos promueven la expansión del proceso de urbanización y con tal de hacer proyectos es posible que recurran a terrenos no aptos para uso residencial. Las autoridades locales deben verificar que un terreno sea apto para los desarrollos, pero al parecer no están logrando ni que se cumpla la ley ni que se respeten las zonas de alto riesgo; los gobernantes de un cantón deben indicar a los inversionistas si existen las condiciones requeridas para que un proyecto pueda ser desarrollado. El cliente o comprador de los bienes inmuebles adquiere las obras sin cuestionamientos y bajo total confianza o simplemente construye en zonas peligrosas por necesidad. Las personas de escasos recursos no tienen alternativa y deciden vivir en zonas no aptas ignorando o conociendo el peligro.

A manera de reflexión, concluimos que el ser humano en general tiende a generar riesgos que no dimensiona en consecuencias que le afectarán a sí mismo. En Costa Rica existen procedimientos adecuados para evitar el desarrollo de asentamientos humanos en zonas de alto riesgo, pero esas normas no están surtiendo efecto y pese a ellas, continúa la construcción de obras civiles en terrenos no aptos. Las autoridades municipales pueden y deben orientar a un inversionista cuando quiera realizar alguna obra en su jurisdicción y el primer instrumento que deben usar para otorgar permiso es el "Uso del Suelo". Si lo solicitado no corresponde con dicho uso, no se debe otorgar el permiso.

Los valles de los ríos deben aparecer como zonas de protección por lo que no debe darse ningún permiso para construir allí. Pero, con o sin permiso, las construcciones están en los valles de los ríos de Moravia. Los inversionistas deben ajustarse a la normativa y abstenerse de construir en sitios inadecuados y evitar que sus posibles clientes corran el riesgo de perder sus bienes y su vida. Quien desee construir o adquirir una vivienda debe preocuparse por el lugar donde hará la inversión y asegurarse de que el sitio no sea riesgoso; deben ir a tanto a la Municipalidad para pedir información y asegurarse que no correrá riesgo viviendo en el sitio elegido. Pero las personas interesadas en comprar o construir una casa no investigan los riesgos del sitio de emplazamiento y terminan viviendo en zonas peligrosas. Una persona sin recurso económico para comprar un lote o una vivienda, se establecerá donde primero pueda, sin importar el riesgo que haya en el sitio escogido. Si los actores sociales mencionados incorporaran la gestión de riesgos en sus planes de vivienda, lo que incluye la prevención del desastre, la obras civiles serían seguras y de bajo riesgo.

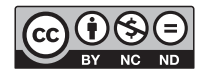

Rev. Reflexiones 98 (1) Enero-Junio, 2019: 7-21, E-ISSN: 1659-2859 
Si el proceso de urbanización formal e informal continúa presionado el uso de la tierra en el cantón de Moravia es muy probable que la gente llegue a estar más cerca de los cauces de ríos y quebradas en el futuro y que las inundaciones aumenten por ello.

Un futuro trabajo debe investigar el estado de la tenencia de la tierra en las zonas invadidas, la condición socioeconómica de las personas que allí habitan y el estado de la educación para la gestión del riesgo en el cantón de Moravia, puesto que la situación anómala demuestra carencias educativas. Por otro lado, las autoridades locales no parecen tener claro que deben reducir la vulnerabilidad humana en vez de fomentarla; el inversionista no parece interesado en la seguridad de sus potenciales compradores y al comprador sólo le interesa tener un techo donde vivir, sin importar si el lugar o la vivienda representan un riesgo para su vida.

\section{Referencias}

Barahona, Dione. (2008). Valoración de la escuela de San Blas afectada por inundación asociada con alcantarillado. DPM-INF-0571-2008. Informe Interno CNE

Bonilla, Esteban. (2007). Inspección en Dos Sectores del Cantón de Moravia, por Problemas Asociados a Laderas Inestables o Erosión Fluvial. DPM-INF-059-2007. Informe Interno CNE

Campos, Alicia. (2011). Valoración del Riesgo por Desprendimiento de Tierra. DPM-INF-0224-2011. Informe Interno CNE.

Chaves, Ignacio. (2010). Valoración del Riesgo de Terreno en La Trinidad. DPM-INF-0936-2010. Informe Interno CNE.

Chaves, Ignacio. (2016a). Valoración de Riesgo de Vivienda por Inundación causada por quebrada y mal manejo de aguas pluviales. IAR-INF-0065-2016. Informe Interno CNE.

Chaves, Ignacio. (2016b). Valoración de Riesgo de Vivienda por Inestabilidad de Ladera y Daños Estructurales. IAR-INF-0315-2016. Informe Técnico CNE.

Méndez, Ileana. (2016). Vivienda en malas condiciones en la ladera del Río Ipís, La Isla de Moravia. GRAE 05-21-04-2016. Informe Técnico CNE.

Sjöbohm, Linda. (2008). Valoración de Viviendas Bajo el Nivel de Carretera y Adyacentes al Río Ipís. DPM-INF-1806-2008. Informe Técnico CNE.

Sjöbohm, Linda. (2009). Valoración de Propiedad Afectada por Erosión Lateral de Quebrada Barreal. DPM-INF-0223-2009. Informe Técnico CNE.

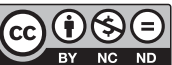


